\title{
Multivariate Genome-Wide Analyses of the Well-being Spectrum
}

Bart M.L. Baselmans ${ }^{1,3}$, Rick Jansen ${ }^{2}$, Hill F. Ip ${ }^{1}$, Jenny van Dongen ${ }^{1,3}$, Abdel Abdellaoui, ${ }^{3,6}$, Margot P. van de Weijer ${ }^{1}$, Yanchun Bao $^{5}$, Melissa Smart ${ }^{5}$, Meena Kumari ${ }^{5}$, Gonneke Willemsen ${ }^{1,3,4}$, Jouke-Jan Hottenga $^{1,3,4}$, BIOS consortium ${ }^{7}$, Social Science Genetic Association Consortium ${ }^{8}$, Dorret I. Boomsma ${ }^{1,3,4}$, Eco J.C. de Geus ${ }^{1,3,4}$, Michel G. Nivard ${ }^{1,3, *}$, Meike Bartels ${ }^{1,3,4, *}$

\section{Affiliations}

${ }^{1}$ Department of Biological Psychology, Vrije Universiteit Amsterdam, Amsterdam, 1081 BT, the Netherlands

${ }^{2}$ Department of Psychiatry, Amsterdam UMC, Vrije Universiteit Amsterdam, the Netherlands, $1081 \mathrm{HL}$, the Netherlands

${ }^{3}$ Amsterdam Public Health Research Institute, Amsterdam UMC, Amsterdam, 1081 BT, the Netherlands

${ }^{4}$ Amsterdam Neuroscience, Amsterdam UMC, Amsterdam, $1081 \mathrm{HV}$, the Netherlands

${ }^{5}$ Institute for Social and Economic Research, University of Essex, Wivenhoe Park, Colchester, Essex, UK

${ }^{6}$ Department of Psychiatry, Amsterdam UMC, University of Amsterdam, the Netherlands

${ }^{7}$ A list of members and affiliations appears in the Supplementary Note.

${ }^{8}$ A list of members and affiliations appears in the Supplementary Note.

* These authors jointly supervised the work and are the corresponding authors

\# These consortia are acknowledged as banner-co-authors for the key role their previous work played. Please find a detailed description of their role and membership at the end of the manuscript

CORRESPONDING AUTHORS: Meike Bartels (m.bartels@vu.nl) and Michel Nivard (m.g.nivard@vu.nl) 
We introduce two novel methods for multivariate genome-wide association meta-analysis (GWAMA) of related traits that correct for sample overlap. A broad range of simulation scenarios supports the added value of our multivariate methods relative to univariate GWAMA. We applied the novel methods to life satisfaction, positive affect, neuroticism, and depressive symptoms, collectively referred to as the well-being spectrum $\left(N_{o b s}=2,370,390\right)$, and found 304 significant independent signals. Our multivariate approaches resulted in a $26 \%$ increase in the number of independent signals relative to the four univariate GWAMA, and in a $\sim 57 \%$ increase in the predictive power of polygenic risk scores. Supporting transcriptome -and methylome-wide analyses (TWAS/MWAS) uncovered an additional 17 and 75 independent loci, respectively. Bioinformatic analyses, based on gene expression in brain tissues and cells, showed that genes differentially expressed in the subiculum and GABAergic interneurons are enriched in their effect on the wellbeing spectrum.

In the past decade, genome-wide association studies (GWAS) have provided insights into the genetic basis of quantitative variation in complex traits ${ }^{1}$. With summary statistics of these GWASs becoming public and the development of linkage disequilibrium score regression (LDSC) ${ }^{2,3}$, genetic correlations between traits can be systematically estimated (e.g. Brainstorm consortium ${ }^{4}$ ). Levering this widely observed genetic overlap between traits, we introduce two novel methods for multivariate genome-wide association metaanalysis, where we define a multivariate model as a model where the effect of a single SNP is considered for multiple traits: 1) N-weighted multivariate GWAMA (N-GWAMA), with a unitary effect of the SNP on all traits, and 2) model averaging GWAMA (MA-GWAMA), where we relaxed the assumption of a unitary effect of the SNP on all traits. Both methods are well equipped to deal with (unknown) sample overlap. The dependence between effect sizes (error correlation) induced by possible sample overlap is estimated from the univariate GWAMA using $\operatorname{LDSC}^{2,3}$. Furthermore, the univariate LDSC intercept is used to correct for population stratification and cryptic relatedness. Both methods have advantages over existing methods. In contrast to MultiPhen ${ }^{5}, \mathrm{CCA}(\mathrm{mv}-\mathrm{PLINK})^{6}$, Combined-PC ${ }^{7}$, and mv-BIMBAM ${ }^{8}$, both our methods can be applied without the need of individual-level genotypic data as only GWAS/GWAMA summary-statistics are required. Additionally, in contrast to $S_{\mathrm{Hom}}{ }^{9}, \mathrm{~N}$-and MA-GWAMA take a more precise estimate of the error correlation into account. In contrast to MTAG ${ }^{10}$, MA-GWAMA, similar to $S_{\text {Het }}{ }^{9}$, generates trait specific estimates for each SNP allowing for a certain degree of heterogeneity (see online methods). Finally, in contrast to TATES ${ }^{11}$, both N-GWAMA and MA-GWAMA generate effect sizes for the multivariate effect where TATES only generates a P-value. The absence of a signed statistic in TATES complicates or even prohibits polygenic prediction. 


\section{Results}

\section{Simulations}

We performed simulations to elucidate in which scenarios N-GWAMA and MA-GWAMA outperform univariate GWAMA, and when N-GWAMA outperforms MA-GWAMA, or the reverse. GWAMA summary statistics were simulated for a range of different scenarios. For each scenario we simulated four heritable traits $\left(h_{\mathrm{SNP}}^{2}=30 \%\right.$ ), and varied the genetic correlation between the four traits from .1 to .9 (see online methods). We sampled 80,000 causal SNPs for 80,000 individuals from the UK Biobank ${ }^{12}$. For each trait, 40,000 individuals were simulated, and the sample overlap between the traits ranged from 0 to 25,000 individuals. On the generated data, four univariate GWAMAs were performed. We chose parameters that far exceed the reported $h_{\text {SNP }}^{2}$ for many complex traits, which allows us to simulate at smaller sample sizes $(\mathrm{N}=80,000)$, reducing the computational burden.

We found that in the presence of genetic correlations equal to, or higher than, .5 , both N-GWAMA and MA-GWAMA outperform univariate GWAMA (Supplementary Fig. 1 and Supplementary Table 1). The added value of multivariate analysis disappears when traits showed lower genetic correlations $(\leq 0.4)$. We performed another four simulation scenarios in which we varied the SNP heritability together with the sample size. We kept the product of the $h_{\text {SNP }}^{2}$ and the sample size constant in order to consider a realistic SNP heritability, which implies that the expectation of the z-statistic remains constant (see online methods). We simulated data with $h_{\mathrm{SNP}}^{2}$ of $40 \%(N=30,000), 20 \%(N=60,000)$, and $10 \%(N=120,000)$, where we kept the genetic correlations between traits constant (at $r_{\mathrm{g}}=0.7$ ). Finally, we simulated four traits with a genetic correlation of $0.7(N=40,000)$, without sample overlap. In all scenarios, both multivariate methods outperformed univariate GWAMA (Supplementary Table 1). To validate MAGWAMA, we simulated data where the assumption of a unitary effects of the SNP on all traits was relaxed (see online methods). We found that, in the scenario where a SNP has an effect on at least three out of four traits, N-GWAMA and MA-GWAMA perform equally well. However, when a SNP has an effect on two out of four traits or one out of four traits, MA-GWAMA outperforms N-GWAMA (Supplementary Table 2). Note that in scenarios where SNPs influence fewer than half of the traits under consideration, univariate methods, such as, GWAMA can outperform multivariate N- and MA-GWAMA.

\section{Application to the well-being spectrum}

Following our longstanding research interest ${ }^{13-16}$, we applied our methods to the following traits; life satisfaction (LS), positive affect (PA), neuroticism (NEU), and depressive symptoms (DS). Despite the fact that the high phenotypic and genetic correlations between these traits is strongly suggestive of a 
common underlying biology, most research is still characterized by separate analyses. Acknowledging this, we performed both N-GWAMA and MA-GWAMA $\left(N_{o b s}=2,370,390\right.$, Supplementary Table 3 $)$ of these four traits to increase the power to identify associated genetic variants.

Our analyses leverage published univariate GWAMAs of life satisfaction ${ }^{15,17}$ ( $N_{\text {obs }}=80,852 ; 2$ studies), positive $\operatorname{affect}^{12,15,17}\left(N_{o b s}=410,603 ; 3\right.$ studies $)$, neuroticism ${ }^{12,15,17,18}$ ( $N_{o b s}=582,989 ; 6$ studies), and depressive symptoms ${ }^{12,15,17,19,20}\left(N_{o b s}=1,295,946 ; 10\right.$ studies $)$. Overall, the mean genetic correlations between different measures of the same trait were higher (LS, $r_{g}=0.68$, PA, $r_{g}=0.9$, NEU, $r_{g}=0.84$, and DS, $\left.r_{g}=0.89\right)$ than the mean genetic correlation between measures of different traits $\left(r_{g}=0.7\right.$; Fig. 1 (upper triangle)). This justifies our two-stage approach of first meta-analyzing the datasets measuring the same traits (LS, PA, NEU, and DS) and secondly meta-analyzing the four resulting datasets into what we refer to as the well-being spectrum $\left(N_{o b s}=2,370,390\right.$; Supplementary Fig. 2). For the purpose of the multivariate GWAMA, we reversed the estimated SNP effects on neuroticism and depressive symptoms to ensure a positive correlation with life satisfaction and positive affect. The dependence between effect sizes (error correlation) induced by sample overlap was estimated from the genome-wide summary statistics obtained from the univariate GWAMA analyses using $\operatorname{LDSC}^{2,3}$ (see online methods and Fig. 1 (lower triangle)). Knowledge of the error correlation between univariate meta-analyses allowed dependent samples to be meta-analyzed, providing a gain in power while guarding against inflated type-1 error rates (see online methods).

\section{Multivariate GWAMA results}

In our N-GWAMA, we identified 231 independent $(250 \mathrm{~kb}$ window LD $>0.1)$ loci associated with the well-being spectrum (Fig. 2A, Supplementary Table 4), whereas MA-GWAMA identified 148 (LS), 191 (PA), 263 (NEU), and 239 (DS) loci (Fig. 2B-E), some of which overlap, resulting in 289 independent signals (Supplementary Table 4 -8). The overlap in genome-wide significant loci divided by the geometric mean of the number of loci discovered for the traits is highly consistent with the genetic correlation between the traits (Supplementary Table 9). Of these 289 independent MA-GWAMA signals, 181 were within a $50 \mathrm{~kb}$ window of the independent signals present in the N-GWAMA analysis (78.3\%). Considering both multivariate methods, we found 304 independent genome-wide signals associated with the well-being spectrum. This is a $26 \%$ increase in comparison to the independent signals found in the univariate GWAMAs (LS, PA, NEU, and DS, Supplementary Table 10 and Supplementary Fig. 3A-D). The low LD-score intercepts for all analyses confirmed that the inflation in test statistics was due to an increase in signal, rather than population stratification or inaccurate accounting for sample overlap (see online methods, Supplementary Table 11). 
We performed a lookup for the genome-wide significant loci reported in published studies of related traits. We identified 26 loci in close proximity $(<250 \mathrm{~kb})$ to the 44 genome wide significant loci $(59.1 \%)$ reported for major depressive disorder $(\mathrm{MDD})^{21}$. In addition, we identified 58 loci in close proximity to the 79 loci identified using an alternative multivariate method considering well-being spectrum traits $(73.4 \%)^{10}$. Using height as a negative control $\left(r_{\mathrm{g}} \sim .05\right.$ with our included traits), we identified 37 loci in close proximity to the 697 loci associated with height $(5.3 \%)^{22}$.

\section{Polygenic prediction}

We compared the predictive power of polygenic scores constructed from univariate GWAMA against NGWAMA and MA-GWAMA. Prediction of measures of LS, PA, NEU, and DS was performed in samples of the Netherlands Twin Register (NTR: ${ }_{\text {mean }} N=>8,100$ ) and Understanding Society (US: ${ }_{\text {mean }} N>8,846$ ) ${ }^{17,23}$. We evaluated the predictive power of each polygenic score by its incremental $\mathrm{R}^{2}$ value, defined as the increase in $\mathrm{R}^{2}$ of the regression including the polygenic score as independent variable together with a set of controls (age, age ${ }^{2}$, sex, and ten principal components) over a regression omitting the polygenic score. Univariate GWAMA polygenic scores had an incremental $\mathrm{R}^{2}$ value of $0.13 \%$ for $\mathrm{LS}, 0.49 \%$ for PA, $1.53 \%$ for NEU, and $1.22 \%$ for DS. The corresponding N-GWAMA and MA-GWAMA had larger incremental $\mathrm{R}^{2}$ (for LS: $0.94 \%$ and $0.92 \%$, for PA: $1.10 \%$ and $1.06 \%$, for NEU: $1.68 \%$ and $1.61 \%$, and for DS: $1.64 \%$ and $1.63 \%$ ). On average, N-GWAMA improved prediction by 59\% and MA-GWAMA improved prediction by 55\% (Supplementary Fig 4 and Supplementary Table 12).

\section{Bioinformatics}

Given the equal performance of both multivariate GWAMAs, and the assumption of TWAS, MWAS, and LDSC that the test statistics follow a standard normal distribution under the null hypothesis of no effect, we have chosen to perform the bioinformatics analyses with the N-GWAMA results.

\section{Transcriptome -and Methylome-wide Analyses}

Multivariate GWAMA aggregates the effect of a single SNP across multiple traits, informed by prior knowledge of the genetic correlation between these traits. Next, we aggregated the effect across multiple SNPs based on prior knowledge that some of these SNPs influence the expression level of a gene transcript or the methylation level at a $\mathrm{CpG}$ site (mQTL) measured in whole blood. These methods (known as TWAS/MWAS) enable identification of genes involved in complex traits ${ }^{24-26}$. In TWAS, we uncovered 97 transcript-trait associations (45 loci) significant at a Bonferroni corrected significance level $\left(p<5.2 \times 10^{-6}\right)$. For 17 TWAS hits (14 loci), the corresponding locus (1000 kb around the transcript) did not contain a significant N-GWAMA SNP. For 49 out of the 97 transcripts (30 loci), the maximum LD 
between the TWAS model SNPs and N-GWAMA top SNP in the corresponding locus was larger than 0.8 (Supplementary Table 13). Furthermore, we found $913 \mathrm{CpG}$ methylation-trait associations mapping to 141 loci at a Bonferroni corrected significance level. For 75 out of $913 \mathrm{CpG}$ methylation-trait associations (36 loci), the corresponding locus did not contain a N-GWAMA significant signal. For $396 \mathrm{CpG}$ methylation-trait associations (83 loci), the maximum LD between the MWAS model SNPs and a NGWAMA top SNP was larger than 0.8 (Supplementary Table 14).

A locus of particular interest was found within the major histocompatibility complex. Recent work has identified 3 individual signals related to schizophrenia (SCZ) in the MHC region, one of which is linked to complement $4(C 4 A)$ gene expression and synapse elimination during puberty ${ }^{27}$. The genome-wide significant signal for the well-being spectrum in the MHC region is not in strong LD with lead eQTL's for $C 4 A$ gene expression. Rather, a second independent signal tagged by rs13194504 is associated with both schizophrenia and well-being. TWAS results for the MHC region implicate the expression of ZKSCAN4 in the etiology of well-being (Supplementary Fig. 5).

\section{Stratified LD Score Regression}

We performed further biological annotation using stratified LD score regression ${ }^{2,3}$. Our first analysis aimed to confirm the involvement of the central nervous system (CNS) in the etiology of the well-being spectrum. Our second analysis aimed to pinpoint specific locations in the brain. Our final analysis used single cell sequencing data to identify specific cell type involvement.

We considered the enrichment in the N-GWAMA derived SNP set of 220 genomic annotations (33 brain and 187 non-brain annotations), which reflected the locations of four specific histone marks (H3K4me1, $\mathrm{H} 3 \mathrm{~K} 4 \mathrm{me} 3, \mathrm{H} 3 \mathrm{~K} 27 \mathrm{ac}$, or H3K9ac) in 54 tissues in their effect on the well-being spectrum ${ }^{28}$. This allows detection of, for example, enrichment of regions of the genome which are histone modified in the prefrontal cortex. Such enrichment would suggest the involvement of processes in the prefrontal cortex in the etiology of the well-being spectrum. Our analyses revealed significant enrichment of 69 annotations characterized by 32 histone marks in 10 brain tissues (Supplementary Table 15 and Supplementary Figure 6). Note that the top 15 significant annotations involve brain tissues. Among these brain tissues are the mid-frontal and inferior-temporal lobe, fetal brain, cingulate and angular gyrus, germinal matrix, hippocampus anterior caudate, substantia nigra, and the neurosphere.

In order to more accurately pinpoint brain regions where genes relevant to the well-being spectrum are differentially expressed, we computed stratified LD scores based on differential gene expression in an anatomically comprehensive set of 210 brain regions, based on 3707 measurements in 6 human brains ${ }^{29}$. 
For each brain region, genes were selected that showed higher expression compared to all other regions (global differential gene expression). The LD scores were significantly enriched at FDR $<0.05$ at multiple gyri in the cortex (Supplementary Table 16). Differential gene expression appeared driven mainly by transcriptional differences between gross anatomical structures in the brain (cortex, sub-cortical structures, brainstem, and cerebellum). To reveal regions related to the well-being spectrum within these structures, we divided the 210 regions into four sets (brain stem, cortex, sub cortex, and cerebellum) based on their locations and computed differential gene expression across the regions within each structure (local differential gene expression).

Our results showed a significant (Bonferroni corrected) enrichment of N-GWAMA signal for genes specifically expressed in the subiculum $(Z=3.47, p<0.001$; Fig. 3A-C and Supplementary Table 1720). The subiculum is considered part of the hippocampal formation and plays a key role in hippocampalcortical interaction $^{30}$, in the inhibition of the Hypothalamic-Pituitary-Adrenal-axis and the human response to stress $^{31}$. We repeated the analyses using GWAMA summary statistics of educational attainment (EA) ${ }^{32}$ and schizophrenia ${ }^{33}$, two traits that are relatively weakly genetically correlated with the well-being spectrum $\left(r_{\mathrm{g}}=-0.15, \mathrm{p}=1.81 \times 10^{-10}\right.$, and $\left.r_{\mathrm{g}}=0.34, \mathrm{p}=2.54 \times 10^{-64}\right)$, but for which the brain has been implicated in their etiologies. In particular, we wanted to see whether the signal observed in the subiculum was specific to the well-being spectrum. As a negative control, we considered the enrichment of genes differentially expressed in all brain regions on height ${ }^{22}$. We found no enrichment of genes differentially expressed in the subiculum on $\mathrm{EA}(\mathrm{Z}=1.251 ; \mathrm{p}=0.105)$, but found an effect on $\mathrm{SCZ}(\mathrm{Z}=2.938 ; \mathrm{p}=$ 0.002). No region was significantly enriched in their effect on height $(p>0.05)$. All results of the differential gene expression analysis were mapped to the MNI coordinates at which the tissue samples were obtained, allowing future integration of our findings and other neuroimaging modalities (Supplementary Table 21 and Fig. 3A-C).

Finally, we obtained the publicly available matrix of gene counts generated based on single nuclei $(N=$ 14,963) from the prefrontal cortex and hippocampus of multiple human donors by Habib et al $(2017)^{34}$. We divided these nuclei into 7 types of neurons, 2 subtypes of astrocytes, oligodendrocytes, oligodendrocyte precursors cells, microglia, endothelial cells, and unclassified cells (hippocampus and prefrontal cortex), and computed cell type specific genes for the different types of neurons (see online methods). Using LDSC we tested the enrichment of all cell-types in the N-GWAMA. Significant enrichment was observed for GABAergic interneurons sampled from the hippocampus and prefrontal cortex $\left(\mathrm{GABA} 1 ; Z=3.42 ; p=3.64 \times 10^{-6}\right.$ and GABA2 $Z=3.7 ; p=6.54 \times 10^{-7}$; Supplementary Table 22 and Fig 3D). 


\section{Discussion}

We have introduced N-GWAMA and MA-GWAMA, two novel methods for conducting multivariate of GWAMA summary statistics of related traits. While previous univariate analyses of traits in the well-being spectrum have been moderately successful, we gained power by the use of multivariate analyses. NGWAMA and MA-GWAMA identified 304 loci associated with some, but not all, traits in the well-being spectrum, and provided flexibility in terms of model specification. Note that model averaging can be extended to incorporate other multivariate models such as $\mathrm{MTAG}^{10}$, or models specified in Genomic $\mathrm{SEM}^{35}$. Model averaging can in fact incorporate any multivariate GWAMA or GWAS model for which the per SNP model fit can be expressed in terms of an AICc fit statistic. Both N-GWAMA and MA-GWAMA are complementary of each other and can thus be used together to identify genetic variants associated with clusters of genetically correlated traits. We illustrate the power gain in multivariate GWAMA over univariate GWAMA for traits genetically correlated above 0.4 using simulations. Empirical application showed that polygenic scores based on multivariate GWAMA outperform polygenic scores based on univariate GWAMA. Besides the advantages of the novel methods, it should be noted that both techniques estimate the error covariance between test statistics from LDSC and therefore inherit the assumptions from LDSC. This implies, that all limitations which apply to LDSC do also apply to our methods. For instance, a previous study found, through simulations, that the univariate LDSC intercept can be biased at very large sample sizes coupled with a high (SNP) heritability ${ }^{36}$.

We used TWAS and MWAS to identify additional loci related to variation in complex traits, like wellbeing, by aggregating the effects across multiple SNPs based on prior knowledge that some of these SNPs influence the expression level of a gene transcript or the methylation level at a $\mathrm{CpG}$ site. By leveraging the N-GWAMA results, LD score regression, and an atlas of brain gene expression, we were able to pinpoint brain regions where region specific gene-expression exists for genes enriched in their effect on well-being. We report evidence for enrichment of genes differentially expressed in the subiculum. Furthermore, we find enrichment for GABAergic interneurons. In the regions for which we have cell types available (hippocampus and prefrontal cortex), we find cell type specific enrichment for the well-being spectrum. However, it stands to reason that the same cell type specific enrichment in other regions may exist, which we may have missed. Gene expression is known to vary systematically between cell-types within the brain $^{37}$ (e.g neurons, microglia, astrocytes) and developmental phases ${ }^{38}$ (prenatally, childhood, adulthood and old age), and likely even between sub-types of a single cell type. Differences in gene expression across or within cell types may induce differences between regions as cell type composition might differ between regions. This limitation needs to be addressed in future well-being research, capitalizing on ongoing efforts to categorize gene expression across the human brain at increased (single cell) resolution. 
Single cell sequencing (e.g. drop-seq based anatomically comprehensive survey of the brain), based on donors deceased at different ages, could disentangle cell-type-specific from region-specific differential gene expression, as well as age specific gene expression ${ }^{39}$. The results of our new multivariate GWAMA methods can be meaningfully mapped to brain regions based on a coordinate system used within multiple other neuroscientific disciplines, facilitating future integration of genetic and neuroscientific research on the well-being spectrum. 


\section{URLs}

N-GWAMA and MA-GWAMA software is available at: https://github.com/

baselmans/multivariate_GWAMA/

TWAS and MWAS software is available at http://bbmri.researchlumc.nl/atlas/\#data;

BIOS consortium https://www.bbmri.nl/acquisition-use-analyze/bios/

TWAS and MWAS prediction models http://bbmri.researchlumc.n1/atlas/\#data

Brain Map gene expression brain regions http://www.brain-map.org/

\section{Acknowledgements:}

We would like to thank all participants in the cohort studies. This work was supported by the Netherlands Organization for Scientific Research (NWO: MagW/ZonMW grants 904-61-090, 985-10-002,904-61193,480-04-004, 400-05-717, NWO-bilateral agreement 463-06-001, NWO-VENI 451-04-034, Addiction31160008, Middelgroot-911-09-032, Spinozapremie 56-464-14192), Biobanking and Biomolecular Resources Research Infrastructure (BBMRI -NL, 184.021.007), the VU University's Institute for Health and Care Research $\left(\mathrm{EMGO}^{+}\right)$and Neuroscience Campus Amsterdam (NCA), the European Science Council (ERC Advanced, 230374), the Avera Institute for Human Genetics, Sioux Falls, South Dakota (USA) and the National Institutes of Health (NIH, R01D0042157-01A). Part of the genotyping was funded by the Genetic Association Information Network (GAIN) of the Foundation for the US National Institutes of Health (NIMH, MH081802) and by the Grand Opportunity grants 1RC2MH089951-01 and 1RC2 MH089995-01 from the NIMH. Part of the analyses were carried out on the Genetic Cluster Computer (http://www.geneticcluster.org), which is financially supported by the Netherlands Scientific Organization (NWO 480-05-003), the Dutch Brain Foundation, and the department of Behavioural and Movement Sciences of the VU University Amsterdam. M. Bartels is/was financially supported by a senior fellowship of the $\left(\mathrm{EMGO}^{+}\right)$Institute for Health and Care and a VU University Research Chair position. This work is supported by an ERC consolidator grant (WELL-BEING 771057 PI Bartels). MGN is supported by a ZonMw grant:" Genetics as a research tool: A natural experiment to elucidate the causal effects of social mobility on health." (pnr: 531003014), ZonMw project: "Can sex- and gender-specific gene expression and epigenetics explain sex-differences in disease prevalence and etiology?" (pnr:849200011) and grant nr: R01AG054628 02S.

Understanding Society is an initiative funded by the Economic and Social Research Council (ES/H029745/1) and various Government Departments, with scientific leadership by the Institute for Social and Economic Research, University of Essex, and survey delivery by NatCen Social Research and Kantar Public. The research data are distributed by the UK Data Service. The genome-wide scan data were analysed and deposited by the Wellcome Trust Sanger Institute. Information on how to access the data can be found on the Understanding Society website https:/www.understandingsociety.ac.uk/. Genotype-trait data access for UKHLS is available by application to Metadac: www.metadac.ac.uk. 


\section{Author Contribution}

M.B., M.G.N. and B.M.L.B. oversaw the study. The theory underlying N-GWAMA and MA-GWAMA was developed by M.G.N. with contribution from B.M.L.B and M.B. Simulations were performed by B.M.LB. and M.G.N. The N-GWAMA and MA-GWAMA software was developed by B.M.L.B., H.F.I., and M.G.N. Data analyses was conducted by B.M.L.B., R. J., H.F.I., J.D., A.A., M.P.W., Y.B., and M.G.N. Data curation was done by R.J., Y.B., M.K., G.W., J.J.H., E.J.C.G., D.I.B., and M.B. Manuscript is written by B.M.L.B., M.G.N., and M.B. with helpful contribution of E.J.C.G. All authors provided input and revisions for the final manuscript.

Competing interests: The authors declare no competing financial interest 


\section{References}

1. Visscher, P. M. et al. 10 Years of GWAS Discovery: Biology, Function, and Translation. Am. J. Hum. Genet. 101, 5-22 (2017).

2. Bulik-Sullivan, B. K. et al. LD Score regression distinguishes confounding from polygenicity in genome-wide association studies. Nat. Genet. 47, 291-295 (2015).

3. Bulik-Sullivan, B. K. et al. An atlas of genetic correlations across human diseases and traits. Nat. Genet. 47, 1236-1241 (2015).

4. Anttila, V. et al. Analysis of shared heritability in common disorders of the brain. Science. 360, 6395 (2018).

5. O'Reilly, P. F. et al. MultiPhen: Joint model of multiple phenotypes can increase discovery in GWAS. PLoS One 7, (2012).

6. Ferreira, M. A. R. \& Purcell, S. M. A. multivariate test of association. Bioinformatics 25, 132-133 (2009).

7. Aschard, H. et al. Maximizing the power of principal-component analysis of correlated phenotypes in genome-wide association studies. Am. J. Hum. Genet. 94, 662-676 (2014).

8. Stephens, M. A. Unified Framework for Association Analysis with Multiple Related Phenotypes. PLoS One 8, (2013).

9. Zhu, X. et al. Meta-analysis of correlated traits via summary statistics from GWASs with an application in hypertension. Am. J. Hum. Genet. 96, 21-36 (2015).

10. Turley, P. et al. Multi-trait analysis of genome-wide association summary statistics using MTAG. Nat. Genet. 50, 229-237 (2018).

11. van der Sluis, S., Posthuma, D. \& Dolan, C. V. TATES: Efficient Multivariate Genotype-Phenotype Analysis for Genome-Wide Association Studies. PLoS Genet. 9, (2013).

12. Bycroft, C. et al. Genome-wide genetic data on $~ 500,000$ UK Biobank participants. bioRxiv 166298, (2017).

13. Bartels, M. \& Boomsma, D. I. Born to be happy? the etiology of Subjective well-being. Behav. Genet. 39, 605-615 (2009).

14. Rietveld, C. A. et al. Molecular genetics and subjective well-being. Proc. Natl. Acad. Sci. U. S. A. 110, 9692-7 (2013).

15. Okbay, A. et al. Genetic variants associated with subjective well-being, depressive symptoms, and neuroticism identified through genome-wide analyses. Nat. Genet. 48, 624-633 (2016).

16. Baselmans, B. M. L \& Bartels, M. A genetic perspective on the relationship between eudaimonic and hedonic well-being. Sci. Rep. 8, 1-10 (2018).

17. Brice, J., Buck, N. \& Prentice-Lane, E. British household panel survey user manual. (1993).

18. Lo, M.-T. et al. Genome-wide analyses for personality traits identify six genomic loci and show correlations with psychiatric disorders. Nat. Genet. 49, 1-8 (2016). 
19. Hyde, C. L. et al. Identification of 15 genetic loci associated with risk of major depression in individuals of European descent. Nat. Genet. 48, 1031-1036 (2016).

20. Hek, K. et al. A genome-wide association study of depressive symptoms. Biol. Psychiatry 73, 667678 (2013).

21. Wray, N. R. et al. Genome-wide association analyses identify 44 risk variants and refine the genetic architecture of major depression. Nat. Genet. 50, 668 (2018).

22. Wood, A. R. et al. Defining the role of common variation in the genomic and biological architecture of adult human height. Nat. Genet. 46, 1173-1186 (2014).

23. Willemsen, G. et al. The Adult Netherlands Twin Register: twenty-five years of survey and biological data collection. Twin Res. Hum. Genet. 16, 271-81 (2013).

24. Zhu, Z. et al. Integration of summary data from GWAS and eQTL studies predicts complex trait gene targets. Nat. Genet. 48, 481-487 (2016).

25. Qi, T. et al. Identifying gene targets for brain-related traits using transcriptomic and methylomic data from blood. bioRxiv 1-26 (2018). doi:10.1101/274472

26. Gusev, A. et al. Integrative approaches for large-scale transcriptome-wide association studies. Nat. Genet. 48, 245-52 (2016).

27. Sekar, A. et al. Schizophrenia risk from complex variation of complement component 4. Nature 530, 177-83 (2016).

28. Finucane, H. K. et al. Partitioning heritability by functional category using GWAS summary statistics. Nat. Genet. 47, 1228-1235 (2015).

29. Hawrylycz, M. J. et al. An anatomically comprehensive atlas of the adult human brain transcriptome. Nature 489, 391-399 (2012).

30. O’Mara, S. M., Commins, S., Anderson, M. \& Gigg, J. The subiculum: A review of form, physiology and function. Prog. Neurobiol. 64, 129-155 (2001).

31. Herman, J. P. \& Mueller, N. K. Role of the ventral subiculum in stress integration. Behav. Brain Res. 174, 215-224 (2006).

32. Okbay, A. et al. Genome-wide association study identifies 74 loci associated with educational attainment. Nature 533, 539-542 (2016).

33. Ripke, S. et al. Biological insights from 108 schizophrenia-associated genetic loci. Nature 511, 421-427 (2014).

34. Habib, N. et al. Massively parallel single-nucleus RNA-seq with DroNc-seq. Nat. Methods 14, 955-958 (2017).

35. Grotzinger, A. D. et al. Genomic SEM Provides Insights into the Multivariate Genetic Architecture of Complex Traits. bioRxiv 305029 (2018). doi:10.1101/305029

36. Vlaming, R. de, Johannesson, M., Magnusson, P. K. E., Ikram, M. A. \& Visscher, P. M. Equivalence of LD-Score Regression and Individual-Level-Data Methods. bioRxiv 211821 (2017). doi:10.1101/211821 
37. Darmanis, S. et al. A survey of human brain transcriptome diversity at the single cell level. Proc. Natl. Acad. Sci. 112, 201507125 (2015).

38. Kang, H. J. et al. Spatio-temporal transcriptome of the human brain. Nature 478, 483-9 (2011).

39. Macosko, E. Z. et al. Highly parallel genome-wide expression profiling of individual cells using nanoliter droplets. Cell 161, 1202-1214 (2015). 


\section{Figure Legends}

Fig 1: Genetic correlations and error correlations (cross-trait intercepts) between the included GWAMA data sets. Upper triangle: genetic correlations. Lower triangle: error correlation representing the magnitude of inflation due to population stratification. Red boxes indicate trait-specific genetic correlations and error correlation. Note, UKB1 represents Caucasian UK Biobank participants living in the UK. UKB2 represents Caucasian UK Biobank participants living in the UK that are relatives from UKB1, and UKB3 represents Caucasian UK Biobank participants not living in the UKB. Sample size of the included traits are displayed in Supplementary Table 3. Note that estimates of genetic correlations as estimated in LDSC are not bounded at 1.

Fig. 2. Manhattan plots of $\mathrm{N}$-weighted and model averaging GWAMA. (a) N-weighted GWAMA. Model averaging GWAMA of (b) life satisfaction, (c) positive affect, (d) neuroticism, (e) depressive symptoms. All plots in all panels are based on the same set of SNPs. The $x$-axis represents the chromosomal position, and the $y$-axis represents the significance on a $-\log _{10}$ scale. Sample size of the included traits are displayed in Supplementary Table 3. Each approximately independent genome-wide significant association ("lead SNP") is marked by $\Delta\left(\mathrm{p}<5 \times 10^{-8}\right)$.

\section{Fig. 3 Local differential gene expression between subcortical structures and enrichment of} individual cell-type enrichment. Local differential gene expression between subcortical structures identifies enrichment of genes specifically expressed in the subiculum $(Z=3.47, p<0.001)$, in their effect on the well-being spectrum. (a) coronial view (b) sagittal view (c) axial view. The location of the samples of brain tissues which were used to measure gene expression by Hawrylycz et al. (2012) is projected to a standard MNI template brain ("Colin27"). The figure is centered on the averaged MNI coordinates of brain samples which are part of the annotation "left Subiculum" ( $x=77, y=90$ and $z=60)$.(d) bar graph representing the cell-type specific enrichment of GABAergic neurons (GABA1; $Z=3.42 ; p=3.64_{\mathrm{X}} 10^{-6}$ and GABA2 $Z=3.7 ; p=6.54_{\times} 10^{-7}$; tested one-sided). Dotted line indicate significant at the FDR $<0.01$ level, dashed line indicate significance at the FDR $<0.05$ level. GABA $=$ GABAergic interneurons, exPFC $=$ glutamatergic neurons from the prefrontal cortex, exCA1/3 = pyramidal neurons from the hippocampus $\mathrm{CA}$ region, $\mathrm{exDG}=$ granule neurons from the hip denate gyrus, $\mathrm{OPC}=$ oligodendrocyte precursors cells, $\mathrm{ASC}=$ astrocytes, $\mathrm{NSC}$, neural stem, cells, $\mathrm{MG}=$ microglia, $\mathrm{ODC} 1=$ oligodendrocytes, endothelian $=$ endothelian cells. N-GWAMA results were used for all analyses $(N o b s=2,370,390)$. 


\section{Online Methods}

\section{N-weighted GWAMA (N-GWAMA)}

We obtained summary statistics from univariate GWAMAs of life satisfaction, positive affect, neuroticism, and depressive symptoms ${ }^{12,15,17-20}$. We used the tool DIST ${ }^{40}$ to impute HapMap2 summary statistics to the 1000Genomes Phase1 reference. To quantify the dependence between the univariate GWAMAs, we estimated the cross trait LD score intercept $(\mathrm{CTI})^{2,3}$ :

$$
C T I=\frac{N_{s} * r_{p}}{\sqrt{N_{1} N_{2}}}
$$

Where $\mathrm{N}_{\mathrm{s}}$ equals the sample overlap, $\mathrm{N}_{1}$ the sample size for trait $1, \mathrm{~N}_{2}$ the sample size for trait 2 , and $r_{p}$ the phenotypic correlation between trait one and two. The CTI is approximately equal to the covariance between the test statistics obtained in univariate GWAMAs of trait 1 and 2. We assume that the estimated CTI is equal to the true CTI, though note the uncertainty in the estimated CTI is generally low. Given the estimated covariance between effect sizes, we can meta-analyse the four dependent GWAMAs and obtain a multivariate test statistic per SNP k:

$$
\mathrm{Z}_{k}=\frac{\sum_{i=1}^{4}\left(w_{i k} * Z_{i k}\right)}{\sqrt{\sum_{i=1}^{4}\left(w_{i k} * V_{i}\right)+\sum_{i=1}^{4} \sum_{j=1}^{4}\left(\sqrt{w_{i k} * w_{j k}} * C_{i, j}\right)(j \neq i)}}
$$

Where $\mathrm{w}_{\mathrm{ik}}$ is the square root of the sample size times the heritability for trait $\mathrm{i}, \mathrm{Z}_{\mathrm{ik}}$ is the test statistic of SNP $\mathrm{k}$ in the GWAMA of trait $\mathrm{i}, \mathrm{V}_{\mathrm{ik}}$ is the variance of the test statistic for SNP $\mathrm{k}$ in the GWAMA of trait $\mathrm{i}$ (i.e 1 given that $\mathrm{Z}$ is a standardized test statistic), and $\mathrm{C}_{\mathrm{i}, \mathrm{j}, \mathrm{k}}$ is the covariance between (standardized) test statistics for SNP $\mathrm{k}$ between GWAMA of trait $\mathrm{i}$ and trait $\mathrm{j}$ (where $\mathrm{C}$ equals CTI obtained from cross trait LD score regression between trait $\mathrm{i}$ and trait $\mathrm{j}$, and $\mathrm{V}$ is the univariate LD score intercept). Under the null hypothesis (no heritability) the test statistics have unit variance and the covariance $\mathrm{C}_{\mathrm{i}, \mathrm{j}}$, is equal to a correlation. The multivariate test statistic $\mathrm{Z}_{k}$, is a weighted sum of tests statistics all of which follow a normal distribution under their respective null distributions. The statistic $\mathrm{Z}_{k}$ follows a standard normal distribution under the null hypothesis of no effect. 


\section{Model averaging GWAMA (MA-GWAMA)}

Consider the following model:

$$
\beta=M V N(\gamma X+e, V)
$$

Where $\beta$ (1xn) is a multivariate normal vector of effect sizes obtained from the regression of $\mathrm{n}$ standardized traits on a standardized genotype (SNP). The matrix V (nxn) is the variance-covariance matrix of effect sizes, matrix $\mathrm{X}$ a design matrix ( $\mathrm{pxn}$ ), and $\gamma$ the corresponding vector of parameters (1xp). The indexed $\mathrm{p}$ denotes the number of variables included in the means model of the response vector $\beta$, and $e$ is the error term.

In this context, a regular GWAMA restricts the design matrix $\mathrm{X}$ to a unit vector (i.e. we model a single genetic effect, which is assumed identical across cohorts, and any observed variation is attributed to sample fluctuation). Generally, matrix $\mathrm{V}$ is diagonal and contains the squared standard errors of elements in $\beta$. A regular GWAMA is the most restricted model one can consider. However, when considering multivariate GWAMA (i.e. the elements in $\beta$ reflect SNP effects on separate yet correlated traits), this model might be too restrictive. Even when traits have a substantial genetic correlation, not all genetic effects need to be shared between traits or be identical in magnitude. The least restrictive model is to consider the SNP effects in $\beta$ independent (i.e. run univariate GWAMA of the correlated traits). In between the most restrictive and least restrictive model, a manifold of models can be specified, equating the effects in $\gamma$ across combinations of traits, while allowing it to differ between other combinations of traits. These models can be specified by ways of the design matrix X.

One could consider a manifold $(\mathrm{z})$ of models $(\mathrm{m})$, each with a different design matrix X.

$$
\begin{aligned}
& \beta_{1}=\operatorname{MVN}\left(\gamma_{1} X_{1}+e, V\right) \\
& \beta_{2}=\operatorname{MVN}\left(\gamma_{2} X_{2}+e, V\right) \\
& \beta_{z}=\operatorname{MVN}\left(\gamma_{z} X_{z}+e, V\right)
\end{aligned}
$$


When considering i correlated traits, a simple expansion of $X$ is to allow for 2 vectors $(p=2)$, a unit vector and a second vector which is coded dichotomously $(0,1)$, where the coding varies over each of the $\mathrm{z}$ models. Other codings, based on analysis of the genetic correlation between traits (i.e. PCA or Cholesky decomposition), can be applied to summary statistics and included in the average. Practically, this allows for the existence of 2 distinct genetic effect. This procedure results in $.5 * p^{2}$ models. The 1-df model with a unit vector for $\mathrm{X}$ and $\left(.5 * p^{2}-1\right)$ 2-df models with a unit vector and a second vector which codes for all possible combinations of pairs of $\mathrm{k}$ traits. However, simply considering m models for all SNPs across the genome results in a prohibitive increase of the already substantial multiple testing burden. Given $\mathrm{z}$ possible models, each of which predict a different vector $\gamma$, and uncertainty for the predicted elements in $\gamma$, a possible way forward is to average the model predictions. The models are weighted by the relative proportion of evidence for each model. Specifically, the weights can be based on the AICc ${ }^{41}$ information criteria. The AICc for model m equals:

$$
A I C c_{m}=-\ln \left(\log L i k_{m}\right)+2 k_{m}+\frac{2 p_{m}\left(p_{m}+1\right)}{z-p_{m}-1}
$$

For each AICc we compute the delta $\left(\Delta_{\mathrm{m}}\right)$ to the best (i.e lowest) AICc value, and from these we compute the model weights $(\mathrm{g})$ for the $\mathrm{p}$ models as:

$$
g_{m}=\frac{\exp \left(-\frac{1}{2} \Delta_{\mathrm{m}}\right)}{\sum_{m=1}^{z} \exp \left(-\frac{1}{2} \Delta_{\mathrm{m}}\right)}
$$

We predict the vector $\beta$ using each of the models

$$
\hat{\beta}_{m}=\gamma_{m} X_{m}
$$

One can aggregate the prediction over all models as: 


$$
\beta_{a}=\sum_{m=1}^{z} \frac{\hat{\beta}_{m} * g_{m}}{\sum_{m=1}^{z} g_{m}}
$$

And we aggregate the uncertainty within and between models to obtain $\operatorname{var}\left(\beta_{a}\right)$ :

$$
\operatorname{var}\left(\beta_{a}\right)=\left[\sum_{\mathrm{m}=1}^{\mathrm{z}} \mathrm{g}_{\mathrm{m}} \sqrt{\operatorname{var}\left(\hat{\beta}_{m}\right)+\left(\hat{\beta}_{m}-\hat{\bar{\beta}}\right)^{2}}\right]^{2}
$$

The resulting vector $\beta_{a}$ contains the model averaged effect sizes for the effect of a particular SNP on the traits subjected to multivariate analysis. Note how the variance estimate contains a variance component which reflects within model variability $\left(\operatorname{var}\left(\hat{\beta}_{m}\right)\right)$ which equals the square of the standard error, and a variance component between model variability $\left(\left(\hat{\beta}_{m}-\hat{\bar{\beta}}\right)^{2}\right)$ in estimate, which ensures no overfitting occurs.

Our procedure boosts power if the SNP effect is concordant between traits, while retaining strongly discordant SNP effects if the model favors these. Model averaging offers several avenues for extension. One can constrain the SNP effects across multiple SNPs based on biological knowledge of the relation between the SNPs and gene expression, or CpG methylation (analog to TWAS). Alternatively, it might be beneficial to average the AICc weights across regions of the genome. Model averaging can in principle accommodate any model for which the AICc information criterion can be expressed. These models should result in a vector of SNP effects $(\beta)$ and an asymptotic variance for the SNP effects. In the current application, models per SNP are estimated in R using the "metafor" package and models are averaged using the "AICcmodavg" package ${ }^{42,43}$.

\section{Simulations}

We performed simulations to elucidate in which scenarios N-GWAMA and MA-GWAMA outperform univariate GWAMA, and when N-GWAMA outperforms MA-GWAMA, or the reverse. For each scenario we simulated four heritable traits $\left(\mathrm{h}_{\mathrm{SNP}}{ }^{2}=30 \%\right)$ effected by $80 \mathrm{~K}$ SNPS. The genetic correlation between the four traits varied between .1 and .9. Using real genotypes and simulated effects, 
We sampled 80,000 causal SNPs for 80,000 individuals from the UK Biobank ${ }^{12}$. For each trait, 40,000 individuals were simulated, in which the sample overlap between the traits ranged from 0 to 25,000 individuals to conducts univariate GWAMA including 656,284 genotyped SNPs (MAF > 0.01). This introduced partial sample overlap between the univariate GWAMA. Next, we performed N-GWAMA and MA-GWAMA analyses and correlated the true SNP effects with the estimated SNP effects obtained from the univariate GWAMA, N-GWAMA, and MA-GWAMA.

We performed another four simulation scenarios in which we varied the SNP heritability together with the sample size. We kept the product of the $h_{\mathrm{SNP}}^{2}$ and the sample size constant in order to test whether at lower $h_{\text {SNP }}^{2}$ and higher sample size (more in line with our empirical application) our findings concerning the relative power of univariate GWAMA, N-GWAMA, and MA-GWAMA persist. The constant product of N $\mathrm{X} h_{\text {SNP }}^{2}$ implies that the expectation of the squared z-statistic remains constant according to the expectation of the square Z statistics as formulated in Bulik-Sullivan et al.2015: (see online methods)

$$
E\left[\chi^{2} \mid \ell_{j}\right]=\frac{N h^{2}}{M} \ell_{j}+N a+1
$$

To validate MA-GWAMA, we simulated data where the assumption of a unitary effect of the SNP on all traits was relaxed. We again simulated four traits, which were affected by $80 \mathrm{~K}$ SNPs. The SNP effects are perfectly correlated, however, we replaced true effects with zero in a way that guarantees that 10K SNPs have a true effect on only one trait, 10K SNPs have a true effect on two traits, and 10K SNPs have a true effect on three traits. Based on these effect sizes and genotypes we simulated traits for $100 \mathrm{~K}$ individuals and performed univariate GWAMA, N-GWAMA, and MA-GWAMA analyses as described above.

\section{Polygenic Prediction}

To confirm the gain in power of our multivariate approaches, we performed polygenic score prediction (PRS) in two independent samples; 1) the Netherlands Twin Register (NTR) 2323,44 and 2) Understanding Society (UKHLS) ${ }^{17}$. We predicted the traits in the well-being spectrum (life satisfaction, positive affect, neuroticism, and depressive symptoms). In NTR, LS and PA data were available in 9,143 and 6,836 genotyped participants, respectively. LS was measured longitudinally using the 5-item Satisfaction with Life Scale ${ }^{45}$. PA is also measured longitudinally using four questions that were adapted from the Subjective Happiness Scale ${ }^{46}$. Neuroticism data were available for 8,527 genotyped participants. The Big Five personality traits (including neuroticism) were measured by using the $\mathrm{NEO}-\mathrm{FFI}^{47}$, a personality questionnaire consisting of five subscales: neuroticism, extraversion, openness, agreeableness and conscientiousness. Depressive symptoms were obtained from the DSM-oriented Depression subscale of the age-appropriate survey from the ASEBA taxonomy ${ }^{48}$ and were available for 7,898 participants. 
In UKHLS data were available for 9,944 participants. LS was measured longitudinally (waves 1-6). Participants were asked how satisfied they were "with life overall", with responses given on a seven-point scale. PA was also measured longitudinally (waves 1 and 4 only) using The Warwick-Edinburgh Mental well-being scale (SWEMWBS) ${ }^{49}$, which is a shortened version of WEMWBS. Neuroticism data were available for 8,198 genotyped participants from wave 3. The Big Five personality traits (including neuroticism) were measured using The Big Five Inventory (BFI), a personality questionnaire consisting of five subscales: neuroticism, extraversion, openness, agreeableness and conscientiousness. Depressive symptoms (DS) were measured longitudinally (waves 1-6) and was obtained from The General Health Questionnaire (GHQ), which was available for 9,203 participants.

The weights used for the polygenic scores were based on the four univariate GWAMAs as well as our two flavors of multivariate GWAMAs. Scores were based on the intersection of SNPs available in any of these GWAMAs and the prediction sample. Both in NTR and UKHLS, SNPs were imputed to 1000 Genomes project March 2012 version $3^{50}$. In NTR, 1,224,793 SNPs and in UKHLS, 955,441 SNPs passed QC, respectively, and were used to construct polygenic scores. The traits were regressed on sex and age as well as principal components included to correct for ancestry, and the polygenic scores. Results can be found in

\section{Supplemental Table 12.}

\section{Summary-Based transcriptome wide (TWAS) association studies}

Gene expression exhibits strong allelic heterogeneity ${ }^{51}$, where multiple SNPs local to the gene jointly influence gene expression levels. We aggregated SNP effects informed by their common effect on expression level of gene (TWAS) or CpG methylation (MWAS), as proposed by Gusev et al. ${ }^{26}$. For TWAS we used the RNA-seq data from the BIOS Consortium ${ }^{52}$. The BIOS Consortium provides a data infrastructure hosting genetic (imputed SNPs), methylome (Illumina 450k array), transcriptome (RNAseq), and phenotypic data on $\sim 4000$ individuals from 6 Dutch biobanks, and a catalog with research output (see URLs). We used 3,344 whole blood RNA-seq samples, measured with Illumina's Hiseq2000 (Pairedend sequencing of $2 \times 50 \mathrm{bp},>15 \mathrm{M}$ read pairs per sample). Batch effects and the first $50 \mathrm{PC}$ 's without a GWAS hit were removed from the RNA-seq data, which was quantile-normal normalized for each gene. The corresponding genotype data we used consisted of 881,977 unambiguous HapMap SNPs (MAF $>5 \%$, minor allele count $>10$, imputation info score $>0.8$ ). For eQTL analysis, linear regression on each SNPgene pair closer than 250k bp was performed. FDR was based on 10 permutations: subject labels were permuted and eQTL analysis was repeated, subsequently top associations per gene were counted and compared between permuted and observed data ${ }^{53}$. At FDR 5\% $(p<1 \mathrm{e}-5)$ there were 13,870 genes with a significant eQTL. For each gene with a significant eQTL, a lasso model was fit in $\mathrm{R}$ with the function 
glmnet, with all SNPs closer than 250k bp to the gene as predictors, and gene expression as outcome. For each gene, Lasso reduces the predictors to an optimal amount and provides the prediction model of gene expression (E) based on local SNPs $\mathrm{S}_{1}-\mathrm{S}_{\mathrm{N}}$ and lasso weights $\mathrm{q}=\mathrm{q}_{1}, \mathrm{q}_{2}, \ldots, \mathrm{q}_{\mathrm{n}}$,

$$
E=\sum_{i=1}^{n} q_{i} S_{i}
$$

Based on the prediction models, N-GWAMA summary statistics and LD based on the 1000 Genomes reference, TWAS was performed. That is, for each gene- prediction-model containing SNPs $\mathrm{S}_{1}-\mathrm{S}_{\mathrm{N}}$ with weights $\mathrm{q}=\mathrm{q}_{1}, \mathrm{q}_{2}, \ldots, \mathrm{q}_{\mathrm{n}}$, the corresponding GWAMA z-scores $\mathrm{z}=\mathrm{z}_{1}, \mathrm{Z}_{2}, \ldots, \mathrm{z}_{\mathrm{n}}$ and LD, an $\mathrm{n}$-by-n correlation matrix for eQTLs $\mathrm{S}_{1}-\mathrm{S}_{\mathrm{N}}$, were used to construct the TWAS test statistic for each gene:

$$
Z_{\text {twas }}=\frac{\sum_{i=1}^{n} q_{i} z_{i}}{\sqrt{q * L D * q}}
$$

TWAS prediction models and LD matrices can be downloaded, and can be used to test for association between the estimated cis (DNA) component of gene expression and the phenotype used for GWAS (see URLs).

\section{Summary-Based methylome wide (MWAS) association studies}

By applying the same approach as TWAS, MWAS was performed using prediction models for each DNA methylation $\mathrm{CpG}$, with local SNPs as predictors. We used whole blood methylation data from the BIOS Consortium $^{54}:$ 4,008 samples measured with Illumina 450K arrays. Methylation pre-processing was the same as for RNA. Genotype and mQTL analysis procedures were the same as for eQTL analysis. At FDR 5\% $(\mathrm{P}<9.3 \mathrm{e}-05)$ there were 151,729 $\mathrm{CpG}$ 's with a significant mQTL. For each $\mathrm{CpG}$ with a significant mQTL we made a prediction model of methylation based on local SNPs (which is a weighted linear combination of SNPs). Based on the prediction models, MWAS statistics were generated (see the formula for TWAS). MWAS prediction models and corresponding LD matrices can be downloaded (see URLs).

\section{Stratified LD score regression}

To determine whether specific genomic regions are enriched for genetic effects on the well-being spectrum, we used LD Score regression ${ }^{2,3}$. We were specifically interested in regions of the genome that are histone modified in a specific tissue. 
We followed the exact procedure described by Finucane et al. $^{28}$, and estimated stratified LD Score regression for the "baseline" model, which contains 53 categories. In addition, we performed analyses using cell type-specific annotations for the four histone marks, corresponding to specific chemical modifications of the histone protein, which packages and orders the DNA molecule. Epigenetic modifications of histones, specifically histones bearing the marks H3K4me1, H3K4me3, H3K27ac or H3K9ac, are associated with increased transcription of DNA into RNA. Each cell type-specific annotation corresponds to a histone mark in a specific cell obtained from distinct human tissue, for example H3K27ac in Fetal Brain cells, generating 220 combinations of histone modification by tissue. When generating estimates of enrichment for the 220 Histone marks by tissue annotations, we controlled for overlap with the functional categories in the full baseline model, but not for overlap with the 219 other cell type specific annotations. For the well-being spectrum, we ran LD Score regression on each of the 220 models (one for each histone by tissue combination) and ranked the histone by tissue annotations by P-value derived from the Z-values of the coefficient. Results are displayed in Supplementary Table 15.

\section{Stratified LD score regression of local gene expression across the human brain.}

We downloaded the normalized and QC'ed gene expression measured in an anatomically comprehensive set of brain regions (see URLs). The data contain 3707 measurements across 6 adult human brains (see Hawrylycz et $\mathrm{al}^{29}$ ). We computed differential gene expression for 48154 probes which map to 20724 unique genes (probes which did not map to genes were omitted). We considered differential gene expression across 210 regions for which at least 3 measurements were available. As Hawrylycz et al. ${ }^{29}$ found little evidence for lateral difference in gene expression, regions in the left and right hemisphere were collapsed into a single region. For each gene in each region a t-test was performed, testing the difference in standardized expression between the region in question and all other brain regions. The top $10 \%$ of probes ranked in terms of t-statistic per region were retained. The unique genes mapped to this set of probes were extracted (mapping 2900-3500 genes to each region). The correlation between t-statistics for the 48,154 probes revealed fairly strong differential expression between the cortex, brainstem, and cerebellum and clustering of differential expression within these regions.

A partitioned LD score with respect to the genomic regions spanned by these genes (using gencode v19 as a reference), and a $100 \mathrm{~kb}$ window around each gene, was computed. The heritability of well-being was partitioned across the 54 baseline annotations ${ }^{28}$ and each of the 210 brain regions (the regions are considered separately). The substantial differences in gene expression between gross anatomical brain regions (cerebellum, cortex, sub-cortical regions and brainstem) dominated the results (Supplementary Table 16). We therefore proceeded to compute differential gene expression within the cerebellum, cortex, sub cortical regions, and brainstem. In this analysis we omitted the fibre bundles as these are anatomically 
distinct from both the cortex and the sub cortical regions, yet not measured densely enough to warrant the computation of differential expression within these fibre bundle tissues. The procedure to compute differentially expressed genes is identical to the procedure used to compute differential expression across the whole brain, but considers the gross anatomical regions separately. New LD scores were computed based on the local differential gene expression analyses (Supplementary Table 17-20). All analyses were repeated using height as a negative control trait. The genomic regions spanning genes differentially expressed in these 210 brain regions were not significantly enriched with SNP effects on height.

\section{Stratified LD score regression of Single nuclei for 7 types of neurons}

We obtained the publicly available matrix of gene counts generated based on single nuclei from the prefrontal cortex and hippocampus of multiple human donors by Habib et al (2017) ${ }^{34}$. To compute differential enrichment we deviated from the procedure outlined for regional brain expression as the zero inflated nature of single nuclei expression violates assumptions of the t-test. The matrix contained counts for 32111 genes measured in 14964 nuclei. The nuclei were divided into 7 types of neurons, 2 subtypes of astrocytes, oligodendrocytes, oligodendrocyte precursors cells, microglia, endothelial cells, and unclassified cells. We omitted genes for which the total count across cells $<150$, or for which less than 30 cells have a count above 0, retaining 11719 genes for analysis. For each gene we computed the ratio of count per nuclei type over the total number of nuclei measured of the specific type (generating the average gene count in each nuclei type). Next, we computed the ratio of the average count per nuclei type over the average count of the gene across all nuclei (generating the nucleic type specific fold change in average expression). We then defined, for each nuclei type, the nuclei type specifically expressed set of genes as the 1600 genes with the highest nucleic type specific fold change in average expression. For each of the gene sets we constructed an LD score with respect to genes in the set, in order to compute the gene set specific enrichment in h2 in our multivariate GWAMA.

Our method to determine cell-type specific expression purely relies on the relative (standardized) expression in one cell type over the (standardized) expression in others, whereas others have developed perhaps more sophisticated statistics to assess differential expression ${ }^{55}$. The test statistics developed by Finucane et al. have never been applied to single cell data, which has a skewed distribution of counts, and we therefore used a simpler metric. Application of both methods to the same gene expression dataset (GTEX) and subsequent differential enrichment analysis for well-being yielded highly correlated enrichment estimates $(\mathrm{r}=.83)$. 


\section{Data Availability}

Summary Statistics excluding results from 23AndMe can be downloaded from

https://surfdrive.surf.nl/files/index.php/s/Ow1qCDpFT421ZOO. The data transfer agreement with 23AnMe stipulates that we can publish effect sizes associated with 10,000 SNPs. These summary statistics can be downloaded from https://surfdrive.surf.nl/files/index.php/s/Ow1qCDpFT421ZOO. For 23AndMe dataset access see: https://research.23andme.com/dataset-access/

\section{Life Sciences Reporting}

Further information on experimental design is available in the Life Science Reporting Summary

\section{Code availability}

N-GWAMA and MA-GWAMA software is available at: https://github.com/ baselmans/multivariate_GWAMA/ 


\section{References}

40. Lee, D., Bigdeli, T. B., Riley, B. P., Fanous, A. H. \& Bacanu, S. A. DIST: Direct imputation of summary statistics for unmeasured SNPs. Bioinformatics 29, 2925-2927 (2013).

41. Akaike, H. A. Bayesian extension of the minimum AIC procedure of autoregressive model fitting. Biometrika 66, 237-242 (1979).

42. Viechtbauer, W. Conducting meta-analyses in R with the metafor package. J. Stat. Softw. 36, 1-48 (2009).

43. Mazerolle, M. J. AICcmodavg: model selection and multimodel inference based on (Q) AIC (c). $R$ Packag. version 1, 1-15 (2011).

44. van Beijsterveldt, C. E. M. et al. The Young Netherlands Twin Register (YNTR): longitudinal twin and family studies in over 70,000 children. Twin Res. Hum. Genet. 16, 252-67 (2013).

45. Diener, E. D., Emmons, R. A., Larsen, R. J. \& Griffin, S. The satisfaction with life scale. J. Pers. Assess. 49, 71-75 (1985).

46. Lyubomirsky, S. \& Lepper, H. S. A measure of subjective happiness: Preliminary reliability and construct validation. Soc. Indic. Res. 46, 137-155 (1999).

47. Costa, P. T. \& McCrae, R. R. Revised NEO personality inventory (NEO PI-R) and NEO five-factor inventory (NEO-FFI) professional manual. (Psychological Assessment Resources Inc., Odessa, 1992).

48. Achenbach, T. M. \& Rescorla, L. Manual for the ASEBA adult forms \& profiles. (Burlington, VT: University of Vermont, 2003).

49. Tennant, R. et al. The Warwick-Dinburgh mental well-being scale (WEMWBS): Development and UK validation. Health Qual. Life Outcomes 5, 1-13 (2007).

50. Altshuler, D. M. et al. An integrated map of genetic variation from 1,092 human genomes. Nature 491, 56-65 (2012).

51. Jansen, R. et al. Conditional eQTL analysis reveals allelic heterogeneity of gene expression. Hum. Mol. Genet. 26, 1444-1451 (2017).

52. Zhernakova, D. V. et al. Identification of context-dependent expression quantitative trait loci in whole blood. Nat. Genet. 49, (2016).

53. Fehrmann, R. S. N. et al. Trans-eqtls reveal that independent genetic variants associated with a complex phenotype converge on intermediate genes, with a major role for the hla. PLoS Genet. 7, (2011).

54. Bonder, M. J. et al. Disease variants alter transcription factor levels and methylation of their binding sites. Nat. Genet. 49, 131-138 (2017).

55. Finucane, H. K. et al. Heritability enrichment of specifically expressed genes identifies diseaserelevant tissues and cell types. Nat. Genet. 50, 621-629 (2018). 